\title{
Suppression of Asporogeny in Bacillus subtilis. Allele-specific Suppression of a Mutation in the spoIIA Locus
}

\author{
By M. D. YUDKIN* AND L. TURLEY \\ Microbiology Unit, Department of Biochemistry, University of Oxford, Oxford $O X 13 Q U$
}

(Received 2 April 1980)

\begin{abstract}
From a strain carrying spoIIA69, a mutation giving rise to asporogeny, a revertant was isolated which sporulated at about $10^{-2}$ of the wild-type frequency but in which the timecourse of sporulation was much protracted. Genetic analysis of this revertant showed that it retained spoIIA69 but had acquired a secondary mutation sas. sas failed to suppress mutations in spoIID, spoIIE or spoIIG; it also failed to suppress another mutation in the spoIIA locus. sas is extremely closely linked (recombination frequency $\leqslant 1 \%$ ) with the mutation spoIIA69 that it specifically suppresses. Strains carrying sas alone sporulated at a frequency at least two orders of magnitude below that in the spoIIA69 sas double mutant. It is suggested that spoIIA69 and sas lead to compensating amino acid changes in the protein specified by the spoIIA locus.
\end{abstract}

\section{INTRODUCTION}

The study of spo mutants has made a most valuable contribution to our understanding of sporulation in Bacillus subtilis. The fact that spo mutations are pleiotropic gives powerful support to the concept that events in sporulation form a dependent sequence (see Mandelstam, 1976), and investigations of the phenotype of mutants blocked at different stages in the sequence have enabled many of these events to be placed in a precise order (Piggot \& Coote, 1976). In spite of intensive work, however, the study of spo mutants has not so far given much information about the means by which the development of spores is regulated.

There have been several reports of the isolation, from spo mutants, of partially revertant strains (work reviewed by Piggot \& Coote, 1976: see also Piggot \& Taylor, 1977; Trowsdale et al., 1979). These strains had regained certain properties associated with the sporulation sequence, but they were still asporogenous.

We have now begun a search for partially suppressed spo mutants (i.e. for strains which, while retaining the spo mutation that gave rise to asporogeny, have acquired a suppressor mutation that now permits them to make some spores) in the hope that a study of such suppressors may help to elucidate the control of spore development. In this paper, we report the isolation of a partially suppressed derivative of a strain carrying the mutation spo- 69 in the spoIIA locus. We show that the new isolate carries a secondary mutation, very closely linked to spoIIA69, but that this suppressor has no effect on any other spo mutation with which we have combined it.

\section{METHODS}

Organisms. These are described in Table 1.

Media. Growth medium and resuspension medium were as described by Sterlini \& Mandelstam (1969). Solid minimal medium was the lactate/glutamate agar of Piggot (1973), but lacking alanine. All of these media were supplemented, when required, with L-cysteine $\left(20 \mu \mathrm{g} \mathrm{ml}^{-1}\right)$, other L-amino acids $\left(50 \mu \mathrm{g} \mathrm{ml}^{-1}\right)$ or uracil $\left(10 \mu \mathrm{g} \mathrm{ml}^{-1}\right)$. In some experiments rifampicin $\left(2 \cdot 5 \mu \mathrm{g} \mathrm{ml}^{-1}\right)$ or sulphanilamide $\left(100 \mu \mathrm{g} \mathrm{ml}^{-1}\right)$ was used. Nutrient agar was from Oxoid. 


\section{Table 1. Bacillus subtilis strains}

In the methods of construction, recipient $\times$ PBS1(donor) $\left(\mathrm{A}^{+}\right)$means that the recipient was transduced with phage PBS1 grown on the donor (Takahashi, 1963); $\mathrm{A}^{+}$recombinants were selected and the desired marker was obtained by cotransduction. Recipient $\times$ DNA(donor) $\left(\mathrm{A}^{+}\right)$means that the recipient was transformed with DNA purified from the donor; $\mathrm{A}^{+}$recombinants were selected and the desired marker was obtained by cotransformation. Recipient $\times$ donor $(b$ by congression with $\mathrm{A}^{+}$) means that the recipient was transformed with a stationary phase culture of the donor (EphratiElizur, 1968); $\mathrm{A}^{+}$recombinants were selected and the desired marker was obtained by congression.

Strain
spo ${ }^{+}$strains
BD112
GSY254
MB10
MB35
168 sul
spo mutants
1
20
55
68
69.2

Strain

MY2001

MY2002

MY2003

MY2007

MY2009

MY2010

MY2011

MY2012

MY2013

MY2014

MY2016

MY2018

MY2019

MY2020

MY2000.01H

MY2000.01L

MY2000.20H

MY2000.20L

MY2000.55

MY2000.68

MY2000.69
Genotype

cys $A$

lys-1 trpC2

pyrD1

hisA aroB2 leuA rfm

$\operatorname{trp} C 2 \mathrm{Sul}^{\mathrm{R}}$
trpC2 spoIIAI
trpC2 spoIIE20
trpC2 spoIIG55
trpC2 spoIID68
metC3 spolla69

\section{Genotype}

hisA pyrD1 aroB2 $\mathrm{rfm}$

hisA pyrDl lys-1 rfm

hisA pyrD1 cys $A$ rfm

metC3 spollA69 sas-1 metC3 spollA69 sas-2 hisA pyrD1 rfm sas-1 lys-1 pyrD1 rfm sas-1 hisA pyrD1 rfm sas-2 lys-1 pyrD1 rfm sas-2 lys-l his $A$ cys $A$ rfm lys-1 hisA rfm Sul ${ }^{\mathrm{R}}$ hisA pyrDI rfm his A pyrD1 rfm spollA69 sas-1 his A pyrD1 rfm spollA69 sas-2 his A pyrD1 rfm spollAI lys-1 pyrDI rfm spoIIAI hisA pyrD1 rfm spoIIE20 lys-1 pyrD1 rfm spoIIE20

\section{lys-1 pyrDI rfm spolIG55} lys-1 pyrD1 rfm spoIID68 lys-1 pyrD1 rfm spollA69

\section{Laboratory stock strains}

Previous designation and reference

\author{
E1 (Piggot, 1973) \\ N25 (Waites et al., 1970) \\ NG12.12 (Piggot, 1973) \\ NG17.29 (Coote \& Mandelstam, 1973) \\ NG18.6 (Piggot, 1973)
}

\section{Constructed strains}

Construction

MB35 $\times$ MB10 (pyrD1 by congression with $\mathrm{Leu}^{+}$) MY2001 $\times$ PBS1(GSY254) $\left(\right.$ Aro $\left.^{+}\right)$

MY2002 $\times$ BD112 (cys $A$ by congression with $\mathrm{Lys}^{+}$) see Results

see Results

MY2002 $\times$ DNA(MY2007) $\left(\mathrm{Lys}^{+}\right)$

MY2010 $\times$ GSY254 (lys- 1 by congression with $\mathrm{His}^{+}$)

MY2002 $\times$ DNA(MY2009) $\left(\mathrm{Lys}^{+}\right)$

MY2012 $\times$ GSY254 (lys-1 by congression with $\mathrm{His}^{+}$) MY2003 $\times$ GSY254 (lys- 1 by congression with $\mathrm{Ura}^{+}$) MY2014 $\times$ PBS1(168 sul) $\left(\right.$ Cys $\left.^{+}\right)$

MY2002 $\times$ DNA(MY2007) $\left(\right.$ Lys $\left.^{+}\right)$

MY2002 $\times$ DNA(MY2007) $\left(\mathrm{Lys}^{+}\right)$

MY2002 $\times$ DNA(MY2009) $\left(\mathrm{Lys}^{+}\right)$

MY2002 $\times 1$ (spoIIAI by congression with Lys $^{+}$)

MY2002 $\times 1$ (spollAI by congression with $\mathrm{His}^{+}$)

MY2003 $\times$ PBS1(20) $\left(\mathrm{Cys}^{+}\right)$

MY2000.20H $\times$ GSY254 (lys- 1 by congression with $\mathrm{His}^{+}$)

MY2002 $\times 55$ (spolIG55 by congression with $\mathrm{His}^{+}$)

MY2002 $\times 68$ (spollD68 by congression with $\mathrm{His}^{+}$)

MY $2002 \times 69.2$ (spoIIA69 by congression with $\mathrm{His}^{+}$)

Sporulation experiments. Bacteria were transferred from growth medium to resuspension medium as described by Sterlini \& Mandelstam (1969). After incubation with shaking (for $15 \mathrm{~h}$ at $37^{\circ} \mathrm{C}$, except where otherwise mentioned), a sample was taken and divided into two portions. One was diluted and plated on nutrient agar; the other was first heated for $30 \mathrm{~min}$ at $85^{\circ} \mathrm{C}$, then diluted if necessary and plated on nutrient agar. Colonies were counted after 18 to $24 \mathrm{~h}$ incubation at $37^{\circ} \mathrm{C}$. The incidence of spores is given as the number of heat-resistant spores per colony-forming unit (c.f.u.).

Transformation. Transformation with stationary phase cultures of the donor was by the technique of Ephrati-Elizur (1968). Transformation with a limiting concentration $\left(0 \cdot 1 \mu \mathrm{g} \mathrm{ml}{ }^{-1}\right)$ of purified DNA [made by the method of Marmur (1961)] was by the technique of Ayad \& Barker (1969). Recombinants were selected on minimal agar with appropriate supplements. Plates were incubated at $37^{\circ} \mathrm{C}$ for $3 \mathrm{~d}$ and then at room temperature for 2 to $4 \mathrm{~d}$. $\mathrm{Spo}^{+}$(sporogenous), $\mathrm{Spo}^{-}$(asporogenous) and various Osp (oligosporogenous) colonies could then be readily distinguished. Spo ${ }^{+}$colonies were brown and opaque, $\mathrm{Spo}^{-} \mathrm{colonics}^{-}$ were colourless and almost transparent, and Osp strains had various intermediate appearances that depended 
on the degree of oligosporogeny. The assignment of colonies to sporulation phenotypes was frequently confirmed by studying their resistance to chloroform vapour. Colonies that had grown on minimal agar in glass plates were replica-plated to nutrient agar, exposed to chloroform vapour at $60^{\circ} \mathrm{C}$ for $30 \mathrm{~min}$, and then again replica-plated to nutrient agar. The two replicas were compared after incubation at $30^{\circ} \mathrm{C}$ for 16 to $24 \mathrm{~h}$. Replicas of $\mathrm{Spo}^{+}$colonies grew equally well on the two plates, $\mathrm{Spo}^{-}$colonies were completely absent from the second plate, and Osp colonies gave various intermediate results depending on the degree of oligosporogeny.

Mutagenesis. (i) With ethyl methanesulphonate. To $5 \mathrm{ml}$ bacteria growing exponentially in growth medium at $37^{\circ} \mathrm{C}$ (about $200 \mu \mathrm{g}$ dry wt $\mathrm{ml}^{-1}$ ) was added $0.1 \mathrm{ml}$ ethyl methanesulphonate (Sigma). After $15 \mathrm{~min}$ incubation with shaking at $37^{\circ} \mathrm{C}$, the bacteria were recovered by centrifugation, washed in warm resuspension medium, and resuspended in $20 \mathrm{ml}$ warm brain heart infusion broth. The culture was shaken at $37^{\circ} \mathrm{C}$ until the bacterial density reached about $250 \mu \mathrm{g} \mathrm{dry} \mathrm{wt} \mathrm{ml}{ }^{-1}$, and the cells were then sedimented by centrifugation and transferred to resuspension medium.

(ii) With ultraviolet irradiation. Cells growing exponentially in $5 \mathrm{ml}$ growth medium at $37^{\circ} \mathrm{C}$ (about

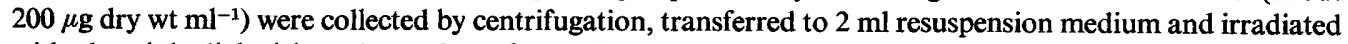
with ultraviolet light (about $3 \mathrm{~J} \mathrm{~m}^{-2} \mathrm{~s}^{-1}$ ) for $2 \mathrm{~min}$. The irradiated suspension was added to $20 \mathrm{ml}$ warm brain heart infusion broth in a foil-covered flask and incubated with shaking at $37^{\circ} \mathrm{C}$. When the bacterial density reached $250 \mu \mathrm{g}$ dry wt $\mathrm{ml}^{-1}$, the cells were sedimented by centrifugation and transferred to resuspension medium.

\section{RESULTS}

\section{Isolation of partial revertants of strain 69.2}

Strain 69. 2 carries spoIIA69, a mutation that blocks sporulation at stage II (Piggot, 1973). When cells harvested from an exponentially growing culture of strain 69.2 were shaken in sporulation salts for about $15 \mathrm{~h}$ at $37^{\circ} \mathrm{C}$ and then heat-treated samples were plated out, colonies arose at a frequency of about 2 per $10^{9}$ c.f.u. All of these appeared to be fully $\mathrm{Spo}^{+}$ revertants, as judged by the appearance of the colonies and by the incidence of spores in a few chosen at random.

In an attempt to find partial revertants, strain 69.2 was treated with ethyl methanesulphonate. Resuspension cultures of mutagenized strain 69.2 contained about eight times as many heat-resistant spores as untreated cultures. About one-quarter of the colonies arising from these spores appeared to be $\mathrm{Spo}^{+}$, but the remainder had the less opaque appearance characteristic of Osp (oligosporogenous) mutants. One of these was picked and purified (MY2007).

In order to isolate another partial revertant, a culture of strain 69.2 was treated with ultraviolet radiation. Resuspension cultures of ultraviolet-treated strain 69.2 contained scarcely any more heat-resistant spores than untreated cultures; however, we did recover one colony of Osp appearance from a heat-treated resuspension culture of irradiated 69.2. This was named MY2009.

On microscopic examination about $8 \mathrm{~h}$ after resuspending MY2007 or MY2009 in sporulation salts, no phase-bright spores were found among more than 1000 cells examined, and only about $10^{-4}$ of the c.f.u. were heat-resistant. In the wild-type, by contrast, most cells contained phase-bright, heat-resistant spores at that time. After $16 \mathrm{~h}$ incubation in resuspension medium, occasional phase-bright spores could be seen in cultures of MY2007 or MY2009, and about $10^{-2}$ of the c.f.u. were heat-resistant, compared with 50 to $80 \%$ in the wild-type. (This point is examined in more detail below.) Strains MY2007 and MY2009 were thus classified as oligosporogenous.

\section{Genetic analysis of strains MY2007 and MY2009}

There are two obvious possible explanations for the oligosporogenous phenotype of MY2007 and MY2009. Either the mutation spoIIA69 has partially reverted, or it has remained unchanged but has been partially suppressed by a second mutation. In the latter case it should be possible to separate the two mutations. 


\section{Table 2. Summary of results of transformation experiments}

DNA purified from $\mathrm{lys}^{+}$donors was used at limiting concentration to transform $l y s$ recipients, and the number of Lys ${ }^{+}$transformants falling into each of four sporulation phenotypes was determined for each cross. 'High Osp' strains gave about 1 spore per $10^{2}$ c.f.u., 'Low Osp' strains about 1 spore per $10^{4}$ to $10^{6}$ c.f.u., and 'Spo'' strains less than 1 spore per $10^{8}$ c.f.u., after $18 \mathrm{~h}$ at $37^{\circ} \mathrm{C}$ in resuspension medium. The percentages given are illustrative; figures such as $70 \%$ and $30 \%$ are not intended to represent the precise result of any particular experiment. Only the sporulation genotypes are given; other markers, omitted in this Table for clarity, are listed in Table 1.

\begin{tabular}{|c|c|c|c|c|c|c|}
\hline \multirow[b]{2}{*}{ Donor strain } & \multirow[b]{2}{*}{ Recipient strain } & \multirow[b]{2}{*}{$\begin{array}{c}\text { Total } \\
\text { Lys }^{+} \\
\text {examined }\end{array}$} & \multicolumn{4}{|c|}{$\begin{array}{l}\text { Approximate frequency of } \\
\text { phenotypic classes among } \\
\text { Lys }^{+} \text {transformants }(\%)\end{array}$} \\
\hline & & & Spo $^{+}$ & $\mathrm{Spo}^{-}$ & $\begin{array}{c}\text { High } \\
\text { Osp }\end{array}$ & $\begin{array}{l}\text { Low } \\
\text { Osp }\end{array}$ \\
\hline 69.2 (spolla69) & MY2002 $\left(s p o^{+}\right)$ & 750 & 70 & 30 & 0 & 0 \\
\hline MY2007 (spollA69 sas-1) & MY2002 (spo+ $)$ & 1720 & 70 & 0 & 30 & $0 \cdot 1$ \\
\hline MY2009 (spollA69 sas-2) & MY2002 (spo+) & 1660 & 70 & 0 & 30 & $0 \cdot 3$ \\
\hline $168 \mathrm{Sul}^{\mathrm{R}}\left(\right.$ spo $\left.^{+}\right)$ & MY2011 (sas-1) & 400 & 30 & 0 & 0 & 70 \\
\hline $168 \mathrm{Sul}^{\mathrm{R}}\left(\mathrm{spo}^{+}\right)$ & MY2013 (sas-2) & 530 & 30 & 0 & 0 & 70 \\
\hline MY2010 (sas-I) & MY2000.69 (spoIIA69) & 12000 & 0 & 70 & $0 \cdot 3$ & 30 \\
\hline MY2012 (sas-2) & MY2000.69 (spoIIA69) & 10000 & 0 & 70 & $0 \cdot 3$ & 30 \\
\hline 69.2 (spoIIA69) & MY2000.69 (spoIIA69) & 15000 & 0 & 100 & 0 & 0 \\
\hline MY2007 (spoIIA69 sas-1) & MY2000.69 (spoIIA69) & 15000 & 0 & 70 & 30 & 0 \\
\hline MY2010 (sas-1) & MY2000.01L (spoIIAI) & 260 & 4 & 67 & 0 & 29 \\
\hline MY2012 (sas-2) & MY2000.01L (spoIIAI) & 420 & 5 & 67 & $\mathbf{0}$ & 28 \\
\hline MY2000.01H (spoIIAI) & MY2011 (sas-1) & 1860 & 0.7 & 30 & 0 & 69 \\
\hline MY2000.01H (spollAI) & MY2013 (sas-2) & 840 & $1 \cdot 5$ & 30 & 0 & 69 \\
\hline MY2012 (sas-2) & MY2011 (sas-1) & 15400 & 0 & 0 & 0 & 100 \\
\hline MY2010 (sas-1) & MY2013 (sas-2) & 17400 & $\mathbf{0}$ & 0 & 0 & 100 \\
\hline
\end{tabular}

spoIIA69 is known to be linked to lys by transformation (Piggot, 1973). When DNA purified from strain 69.2 was used to transform strain MY2002 $\left(s p o^{+} l y s\right)$, about one-third ( 27 to $36 \%$ ) of the Lys ${ }^{+}$transformants were Spo- ${ }^{-}$. When purified DNA from strain MY2007 was used to transform strain MY2002, about two-thirds of the Lys ${ }^{+}$transformants were $\mathrm{Spo}^{+}$and one-third resembled MY2007; however, two out of 1720 transformants had a more translucent, colourless appearance. Similarly, when strain MY2002 was transformed with DNA from MY2009, five out of 1660 transformants were more translucent and colourless than is characteristic of strain MY2009. (The results of these and subsequent transformations are summarized in Table 2.)

We initially suspected that this minority class of seven transformants carried spoIIA69 only, separated by recombination from a suppressing mutation, but subsequent work disproved this belief. When streaked on plates of minimal agar and incubated for $3 \mathrm{~d}$ at $37^{\circ} \mathrm{C}$, these transformants appeared more opaque than strain MY2000.69 (an isogenic strain carrying spoIIA69) but less opaque than MY2007 or MY2009, which suggested that they represented a class that produced spores at a low frequency, but higher than that of strains carrying spoIIA69 alone. In fact, when tested in resuspension experiments (in whicb MY2007 and MY2009 gave heat-resistant spores at a frequency of about 1 per $10^{2}$ c.f.u. and MY2000.69 at a frequency of less than 1 per $10^{8}$ c.f.u.) all seven of these transformants gave heat-resistant spores at a frequency of 1 per $10^{4}$ to $10^{6} \mathrm{c}$.f.u. These spores themselves gave rise to similar oligosporogenous populations. Repetition of these experiments showed that such variability of about two orders of magnitude in the frequency of appearance of spores was characteristic of these strains: there was no evidence that the seven transformants belonged to more than one class. The sporulation phenotype of the strains just described, and of others mentioned below, is given in Table 3 .

Our interpretation of these facts is as follows. Strains MY2007 and MY2009 arose by the 


\section{Table 3. Incidence of spores in resuspension cultures of mutant strains}

\section{The numbers of heat-resistant spores and of total c.f.u. were determined after $18 \mathrm{~h}$ at $37^{\circ} \mathrm{C}$ in} resuspension medium. The incidence of spores is given to the nearest power of 10 .

Strain
69.2
MY2007
MY2009
MY2010, MY2011
MY2012, MY2013
MY2019
MY2020
MY2000.01H, MY2000.01L
MY2000.69

Sporulation genotype
spollA69
spoIIA69 sas-1
spoIIA69 sas-2
sas-1
sas-2
spoIIA69 sas-1
spoIIA69 sas-2
spoIIA1
spoIIA69

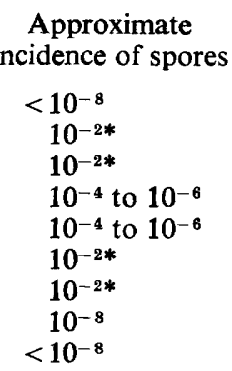

* The time-course of sporulation in these strains was very protracted.

acquisition of secondary mutations by strain 69.2 . These secondary mutations partially suppress the asporogenous mutation spoll A69. We call the secondary mutation in MY2007 sas-1 (for suppression of asporogeny) and that in MY2009 sas-2. Mutations sas-1 and sas-2 are both very closely linked to spoIIA69, but they can be separated from it at a low frequency. The rare class among the Lys ${ }^{+}$transformants produced when MY2002 was transformed with DNA from MY2007 or MY2009 contain only sas, separated from spoIIA69; because recombinants carrying only spoIIA69 were not recovered in these crosses, sas -1 and sas-2 probably lie between spoIIA69 and lys. Strains carrying sas-1 (MY2010) or sas-2 (MY2012) unaccompanied by any other spo mutation sporulate at a rather low but variable frequency; hence sas- 1 and sas- 2 are themselves spo mutations.

\section{Phenotypes of strains carrying sas mutations}

When cultures of MY2000.69 that had been incubated in resuspension medium for several hours at $37^{\circ} \mathrm{C}$ were examined by light microscopy, most of the cells had an abortively disporic appearance. This phenotype is characteristic of strains carrying mutations at the spoIIA locus (Piggot \& Coote, 1976). Resuspension cultures of the sas mutants MY2010 and MY2012 were indistinguishable in microscopic appearance from MY2000.69, which tends to bear out our conclusion that the sas mutations are, in reality, mutations in spoIIA, very closely linked to spoIIA69. Cells taken from resuspension cultures of the double mutants MY2019 and MY2020 (Table 1) were again indistinguishable.

Although the incidence of heat-resistant spores in resuspension cultures of MY2010 (sas-1) and MY2012 (sas-2) was quite variable, the incidence in corresponding cultures of MY2019 (spoIIA69 sas-1) and MY2020 (spoIIA69 sas-2) was more constant. On several occasions the number of heat-resistant spores finally obtained in resuspension cultures of MY2019 and MY2020 was between 5 per $10^{3}$ c.f.u. and 2 per $10^{2}$ c.f.u. However, the time-course of sporulation in these strains was very protracted (Fig. 1). Heat-resistant spores appeared in a resuspension culture of MY2020 several hours later than in a similar $s p o^{+}$ culture (strain MY2019 was indistinguishable from MY2020). By contrast, the time-course of appearance of heat-resistant spores in MY2010 (sas-1) and MY2012 (sas-2) resembled that of the spo $^{+}$strain MY2018.

We wondered whether strains MY2019 and MY2020 might be temperature-sensitive for sporulation, but found that the number of spores finally obtained from these strains was no different at 30,37 or $41{ }^{\circ} \mathrm{C}$. 


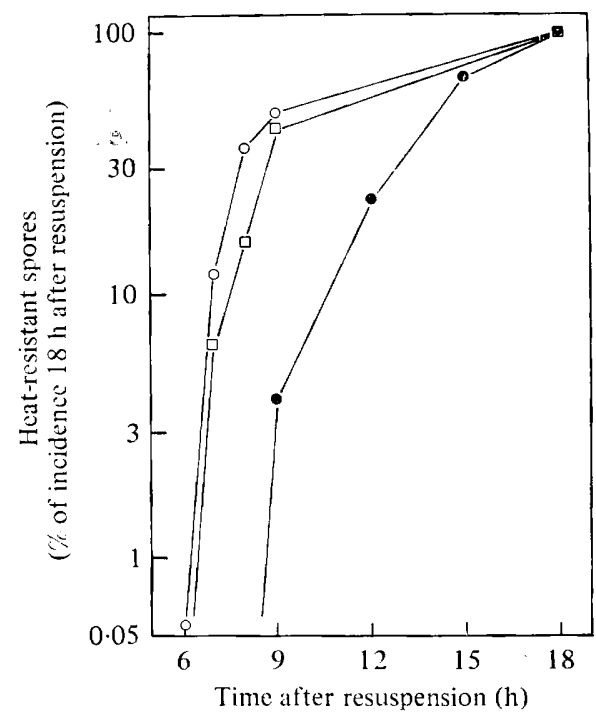

Fig. 1. Time-course of appearance of heat-resistant spores in strains MY2012, MY2018 and MY2020. Cells from exponentially growing cultures of MY2012 (sas-2) ( $\square$ ), MY2018 (spo ${ }^{+}$) (O) and MY2020 (spoIIA69 sas-2) (O) were resuspended in sporulation medium. At intervals, samples were taken and the numbers of heat-resistant spores and of total c.f.u. were found. The incidence of heat-resistant spores is plotted as a percentage of the incidence $18 \mathrm{~h}$ after resuspension.

\section{Genetic analysis of sas mutations}

To test our conclusion that sas-1 and sas-2 lie in spoIIA, we transformed MY2011 (lys sas-1) and MY2013 (lys sas-2) with DNA from a wild-type. Among the Lys ${ }^{+}$transformants about one-third ( 25 to $37 \%$ ) were $\mathrm{Spo}^{+}$.

We have suggested that sas- 1 and sas- 2 are mutations that are closely linked to spoIIA69 and partially suppress the asporogeny imposed by the latter, and that MY2010 and MY2012 carry sas mutations unaccompanied by other spo mutations. If these suggestions are correct, it should be possible to cross MY2000.69 with MY2010 or MY2012 and recover recombinants that resemble the original isolates MY2007 and MY2009.

We transformed MY2000.69 with DNA purified from MY2010. Out of about 12000 Lys $^{+}$transformants, 33 appeared substantially more opaque and pigmented than the remainder, although readily distinguishable, both by the appearance of the colonies and by their sensitivity to chloroform vapour, from $\mathrm{Spo}^{+}$strains. We chose six of these at random and tested their frequency of sporulation in a resuspension experiment. After $18 \mathrm{~h}$ in resuspension medium the number of heat-resistant spores in the six cultures ranged from 4 to 15 per $10^{3}$ c.f.u. This frequency of spores is very similar to that in MY2019, which (if our interpretation is correct) has the same sporulation genotype (spoIIA69 sas-1).

In a similar experiment, we recovered 27 pigmented colonies from about $10000 \mathrm{Lys}^{+}$recombinants when we transformed MY2000.69 with DNA from MY2012. In five of these chosen at random the frequency of heat-resistant spores after $18 \mathrm{~h}$ in resuspension medium was 5 to 10 per $10^{3}$ c.f.u.

The fact that spoIIA69 sas double mutants were recovered in these experiments, but spo ${ }^{+}$ recombinants were not, supports our belief that the order of the markers is spo-69. .sas ...lys. Given this order, $s p o^{+}$recombinants could only have been produced in these transformations by quadruple crossovers.

The results can be used to obtain the recombination frequency between spo-69 and sas. When we transformed MY2002 with the DNA from MY2010, $67 \%$ of the Lys ${ }^{+}$transformants were $\mathrm{Spo}^{+}$. Hence, although $12000 \mathrm{Lys}^{+}$recombinants were obtained when we trans- 
formed MY2000.69 with the same DNA preparation, in 8000 of these the sas-1 marker will not have been available for recombination with spoIIA69 in the recipient. Of the remaining 4000 transformants, 33 were spo-69 sas- 1 double mutants; thus, the recombination frequency between the markers is $0.8 \%$. A similar calculation gives a recombination frequency of $0.7 \%$ between spo- 69 and sas-2.

In control experiments, we transformed MY2000.69 with DNA from 69.2 or from MY2007. Out of more than 15000 Lys $^{+}$transformants from each of these crosses, none was $\mathrm{Spo}^{+}$(see Table 2).

\section{Effect of sas mutations on strains carrying mutations in other spoII loci}

To see whether sas-1 and sas-2 suppressed other spoII mutations, rather than spoIIA69 specifically, lys strains carrying spoIID68, spoIIE20 or spoIIG55 (see Table 1) were each transformed with DNA from MY2010 or MY2012. About one-third of the Lys ${ }^{+}$transformants in such experiments carried sas, so if sas mutations suppressed the other spo markers one would have expected one-third of these transformants to show a $\mathrm{Spo}^{+}$(or at least Osp) phenotype. In fact, out of several hundred recombinants from each transformation more than $99 \%$ remained $\mathrm{Spo}^{-}$, as shown both by the absence of pigment and by their extreme sensitivity to chloroform vapour.

\section{sas mutations do not suppress spollal}

We wanted to determine whether sas mutations were capable of suppressing a mutation in spoIIA other than spoIIA69. When MY2000.01L (spoIIAI lys) was transformed with DNA from MY2010 (sas-1 lys ${ }^{+}$), about two-thirds of the Lys ${ }^{+}$transformants resembled strains carrying spoIIA1 (Spo-) and one-third resembled MY2010 (Osp), but 10 out of 260 Lys $^{+}$transformants were more pigmented than the latter (Table 2). If sas-1 did in fact suppress spoIIA1, one would have expected that among these pigmented colonies there would have been some that carried the two mutations spoIIA and sas-1. However, this argument is sound only if the order of the markers is spoIIA1. . sas -1 . . lys. If the order of the markers were sas-1...spoIIA1...lys, then the large majority of pigmented colonies in the above cross would be $s p o^{+}$, even if the double mutant spoIIA1 sas-1 were pigmented. We therefore made the reciprocal cross by transforming MY2011 (sas-1 lys) with DNA purified from MY2000.01H (spoIIA1 lys $\mathrm{s}^{+}$). Out of $1860 \mathrm{Lys}^{+}$transformants, 14 were more pigmented than MY2011; 10 of these were picked at random and purified.

We were now sure that, whatever the order of markers, among the 20 pigmented colonies picked from the two reciprocal crosses there would be several that carried both spoIIAI and sas-1, if this double mutant was more pigmented than strains carrying sas- 1 alone.

All of these 20 pigmented recombinants seemed, by their appearance and their resistance to chloroform vapour, to be $\mathrm{Spo}^{+}$, but, to be sure that none of them carried both sas- 1 and spoIIA1, each of them was used as a donor in transformation by the technique of EphratiElizur (1968). The recipient was MY2016 (lys his A Sul ${ }^{\mathrm{R}}$ ), and Lys ${ }^{+}$recombinants were selected on minimal plates containing histidine and sulphanilamide. If in such a cross the donor carried the two spo mutations spoIIA1 and sas-1, then these mutations (which, as the above results show, recombine quite readily) would sometimes be separated. The result would be that a few per cent of the Lys ${ }^{+}$transformants would be of Spo- or Osp appearance.

We examined at least $500 \mathrm{Lys}^{+}$transformants from each of the 20 experiments, but in no case was there evidence that the donor contained a spo mutation linked to lys. (In 15 of the 20 cases all of the transformants were $\mathrm{Spo}^{+}$: in the remaining five cases about $10^{-3}$ of all the $\mathrm{Lys}^{+}$transformants were $\mathrm{Spo}^{-}$, but when these were analysed they all resembled strains carrying spoO mutations. In no case was the spo mutation linked by transformation to lys; we suspect that a very few transformants had adventitiously acquired spoO mutations.) This entire experiment was repeated by making the two analogous reciprocal crosses between 
strains carrying spoIIAI and strains carrying sas-2 and picking pigmented colonies; the results were similar (Table 2 ).

We concluded that all the pigmented colonies which emerged from crosses between spoIIAI and sas were $s \mathrm{Po}^{+}$, and therefore that the doubly mutant recombinants spoIIAI sas were not pigmented. Since in these recombination experiments we had been careful to pick all colonies that were more pigmented than strains carrying sas alone (and suppressed strains carrying spoIIA69 together with sas could easily be recognized as more pigmented than those carrying sas alone), it appeared that sas mutations did not suppress spoIIAI.

The results of these experiments were used to derive a probable order of the markers spoIIAl, sas- 1 and lys. With sas-1lys ${ }^{+}$as donor and spoIIAl|lys as recipient, 10 out of $260 l y s^{+}$ transformants $(3.8 \%)$ were $s p o^{+}$, but in the reciprocal cross with spoIIAl lys ${ }^{+}$as donor and sas- 1 lys as recipient, only 14 out of $1860 \mathrm{lys}^{+}$transformants $(0.75 \%)$ were $s p o^{+}$. If we assume that in the latter case $s p o^{+}$recombinants had to be generated by quadruple crossovers, the order is sas-1...spo-1...lys. The results of the crosses between sas-2 and spoIIA1 similarly suggest the order sas-2. . spo-1. . .lys (Table 2).

\section{Attempts to recombine sas-1 and sas-2}

Although MY2007 (spoIIA69 sas-1) and MY2009 (spoIIA69 sas-2) were isolated independently, they were extremely similar both in their genetic behaviour and in their phenotypic effects and it seemed possible that they were, in fact, the same mutation. If they were different, one might have been able to recombine them to generate $s p o^{+}$.

We transformed MY2011 (sas-1 lys) with DNA from MY2012 (sas-2 lys ${ }^{+}$) and obtained 15400 Lys $^{+}$recombinants, and we transformed MY2013 (sas-2 lys) with DNA from MY2010 (sas-1 lys ${ }^{+}$) and obtained $17400 \mathrm{Lys}^{+}$recombinants. All these recombinants were carefully examined; every one retained the Osp phenotype of the parents. We therefore suspect that sas-1 and sas-2 are identical.

\section{DISCUSSION}

We interpret our results as follows. sas is a mutation in spoIIA which, when unaccompanied by spollA69, renders strains oligosporogenous. Such strains have, in resuspension cultures, the typical appearance of spoIIA mutants. sas does not suppress mutations in three other spo loci that we have tested [one of which immediately precedes spoIIA in the dependent sequence (Piggot \& Coote, 1976)], and it does not suppress spoIIA1. Had sas been discovered during a routine search for spo mutations it would have seemed a run-of-themill spoIIA mutation.

Coote (1972) has produced evidence suggesting that a mutation leading to oligosporogeny can occur in the same gene as a mutation leading to asporogeny. Our results point to the same conclusion: spoIIA69 and sas are very closely linked (recombination frequency $\leqslant 1 \%$ ), and although it is naturally possible that the mutations are near the ends of contiguous genes it seems more likely that they lie in the same gene. Moreover, we have shown that the probable order of mutations is spo-69...sas. . spo-1...lys, which would place a mutation giving oligosporogeny between two mutations giving asporogeny.

What is remarkable about sas is that it partially suppresses spoIIA69, a mutation which renders strains asporogenous. One might also say, with some justification, that spoIIA69 suppresses sas: the frequency of spores produced in resuspension cultures of spoIIA69 sas double mutants is at least two orders of magnitude higher than in mutants containing sas alone. This fact seems important in our understanding of how sas works.

We have considered a model in which spoIIA69 and sas are both promoter mutations; when both mutations are present promoter activity is higher than with either alone. However, the combination of spoIIA69 and sas, although it ultimately leads to the production of more spores than are found in the single mutants, renders sporulation unusually pro- 
tracted; moreover, spoIIA1 produces a phenotype indistinguishable from that of spoIIA69 mutants (unpublished results), and yet the recombination frequency between sas and spoIIA seems much too high for spoIIAI also to lie in the same promoter.

A more likely possibility is that spoIIA69 is a chain-terminating mutation and sas a mutation that reinitiates translation (Grodzicker \& Zipser, 1968). Here again, however, the sporulation properties of strains carrying the separate mutations or the two together, although not incompatible with such a model, are not what one would have predicted from it. Moreover, spoIIA69, although a point mutation, is not suppressed by the nonsense suppressor $\mathrm{su}^{+} 3$ (unpublished results). We have also established that $s a s-1$ does not suppress the amber mutations metB5 or purB6 (results not shown).

The explanation that we favour is that spoIIA encodes a protein that is essential for sporulation, that spoIIA69 produces an amino acid replacement that abolishes activity, and that sas, when present alone, produces a replacement that renders the protein very weakly active. However, the two amino acid changes compensate, with the result that a strain carrying both mutations sporulates at a higher frequency than strains carrying either alone. [Compensating amino acid changes are well known in other systems (Helinski \& Yanofsky, 1963)]. To verify this explanation one would need to isolate the product of the spoIIA gene.

Whether this model is correct or not, it seems clear that each of three different mutant forms of spoIIA leads to a characteristic sporulation phenotype: spoIIA69 alone gives asporogeny; sas alone gives low oligosporogeny $\left(10^{-6}\right.$ to $\left.10^{-4}\right)$; spoIIA69 and sas together give high oligosporogeny (about $10^{-2}$ ) with sluggish production of spores. These results suggest the possibility that spoIIA may have an important role in the regulation of sporulation. Further study of the mutants described here, and in particular a detailed investigation of the temporal sequence of events during sporulation of spoIIA69 sas double mutants (whose delayed sporulation is not immediately explicable in terms of any of the models we have mentioned), might throw some light on the control of spore development.

We are greatly indebted to Dr B. N. Dancer, Professor J. Mandelstam and Dr P. J. Piggot for their advice and for commenting on an earlier draft of the manuscript.

\section{REFERENCES}

AYAD, S. R. \& BARKER, G. R. (1969). The integration of donor and recipient deoxyribonucleic acid during transformation of Bacillus subtilis. Biochemical Journal 113, 167-174.

Coоте, J. G. (1972). Sporulation in Bacillus subtilis. Genetic analysis of oligosporogenous mutants. Journal of General Microbiology 71, 17-27.

Coote, J. G. \& Mandelstam, J. (1973). Use of constructed double mutants for determining the temporal order of expression of sporulation genes in Bacillus subtilis. Journal of Bacteriology 114, 1254-1263.

Ephrati-Elizur, E. (1968). Spontaneous transformation in Bacillus subtilis. Genetical Research 11, 83-96.

GrodzICKER, T. \& ZIPSER, D. (1968). A mutation which creates a new site for the re-initiation of polypeptide synthesis in the $Z$ gene of the lac operon of Escherichia coli. Journal of Molecular Biology 38, 305-314.

Helinski, D. R. \& Yanofsky, C. (1963). A genetic and biochemical analysis of second site reversion. Journal of Biological Chemistry 238, 1043-1048.

MANDElstaM, J. (1976). Bacterial sporulation: a problem in the biochemistry and genetics of a primitive developmental system. Proceedings of the Royal Society of London B 193, 89-106.

MARMUR, J. (1961). A procedure for the isolation of deoxyribonucleic acid from micro-organisms. Journal of Molecular Biology 3, 208-218.

Piggot, P. J. (1973). Mapping of asporogenous mutations of Bacillus subtilis: a minimum estimate of the number of sporulation operons. Journal of Bacteriology 114, 1241-1253.

Piggot, P. J. \& Coote, J. G. (1976). Genetic aspects of bacterial endospore formation. Bacteriological Reviews 40, 908-962.

Piggot, P. J. \& TAYlor, S. Y. (1977). New types of mutation affecting formation of alkaline phosphatase by Bacillus subtilis in sporulation conditions. Journal of General Microbiology 102, 69-80.

Sterlini, J. M. \& Mandelstam, J. (1969). Commitment to sporulation in Bacillus subtilis and its relationship to development of actinomycin resistance. Biochemical Journal 113, 29-37.

TAKAHASHI, I. (1963). Transducing phages for Bacillus subtilis. Journal of General Microbiology 31, 211-217.

Trowsdale, J., Chen, S. M. H. \& Hoch, J. A. (1979). Genetic analysis of a class of polymixin 
resistant partial revertants of stage $O$ sporulation mutants of Bacillus subtilis: map of the chromosome region near the origin of replication. Molecular and General Genetics 173, 61-70.

Waites, W. M., Kay, D., Dawes, I. W., Wood,
D. A., Warren, S. C. \& Mandelstam, J. (1970). Sporulation in Bacillus subtilis. Correlation of biochemical events with morphological changes in asporogenous mutants. Biochemical Journal 118, $667-676$. 